\title{
Revised and edge revised Szeged indices of graphs
}

\author{
Morteza Faghani, Ali Reza Ashrafi * \\ Department of Mathematics, Faculty of Mathematical Science, \\ University of Kashan, Kashan 87317-51167, I. R. Iran
}

Received 31 October 2011, accepted 19 August 2012, published online 8 April 2013

\begin{abstract}
The revised Szeged index is a molecular structure descriptor equal to the sum of products $\left[n_{u}(e)+n_{0}(e) / 2\right] \times\left[n_{v}(e)+n_{0}(e) / 2\right]$ over all edges $e=u v$ of the molecular graph $G$, where $n_{0}(e)$ is the number of vertices equidistant from $u$ and $v, n_{u}(e)$ is the number of vertices whose distance to vertex $u$ is smaller than the distance to vertex $v$ and $n_{v}(e)$ is defined analogously. In this paper, new formula for computing this molecular descriptor is presented by which it is possible to reprove most of results given in [M. Aouchiche and P. Hansen, On a conjecture about the Szeged index, European J. Combin. 31 (2010), 16621666]. We also present an edge version of this graph invariant. At the end of the paper an open question is presented.
\end{abstract}

Keywords: Szeged index, edge Szeged index, revised Szeged index, edge revised Szeged index.

Math. Subj. Class.: $05 C 12$

\section{Introduction}

We first describe some notations which will be kept throughout. Let $G$ be a simple graph with vertex set $V(G)$ and edge set $E(G)$. If $e=u v \in E(G)$ then $d(u, v)$ stands for the distance between $u$ and $v$ in $G$. A topological index is a graph invariant applicable in chemistry. A topological index $\chi$ is called distanced-based, if $\chi$ is related to the distance function $d(-,-)$. The first use of a distance-based topological index occurred in the year 1947 in a seminal paper by an American chemist Harold Wiener [14].

Suppose $G$ is a connected graph and $e=u v \in E(G)$. The quantities $n_{0}(e), n_{u}(e)$ and $n_{v}(e)$ are defined to be the number of vertices equidistant from $u$ and $v$, the number of vertices whose distance to vertex $u$ is smaller than the distance to vertex $v$ and the number

* Corresponding author.

E-mail addresses: m_faghani@pnu.ac.ir (Morteza Faghani), ashrafi@kashanu.ac.ir (Ali Reza Ashrafi) 
of vertices closer to $v$ than $u$, respectively. Similarly, the quantities $m_{0}(e), m_{u}(e)$ and $m_{v}(e)$ are defined to be the number of edges equidistant from $u$ and $v$, the number of edges whose distance to vertex $u$ is smaller than the distance to vertex $v$ and the number of edges closer to $v$ than $u$, respectively. Here, for an edge $e=x y$ and vertex $u$, the distance between $e$ and $u$ is defined as $d_{G}(e, u)=\operatorname{Min}\left\{d_{G}(x, u), d_{G}(y, u)\right\}$.

The Szeged, edge Szeged, edge-vertex Szeged, vertex-edge Szeged, revised Szeged and edge revised Szeged indices of $G$ are defined as follows:

$$
\begin{aligned}
S z(G) & =\sum_{e=u v}\left[n_{u}(e) \times n_{v}(e)\right] \\
S z_{e}(G) & =\sum_{e=u v}\left[m_{u}(e) \times m_{v}(e)\right] \\
S z_{e v}(G) & =1 / 2 \sum_{e=u v}\left[m_{u}(e) \times n_{v}(e)+m_{v}(e) \times n_{u}(e)\right], \\
S z_{v e}(G) & =1 / 2 \sum_{e=u v}\left[m_{u}(e) \times n_{u}(e)+m_{v}(e) \times n_{v}(e)\right], \\
S z^{*}(G) & =\sum_{e=u v}\left[\left(n_{u}(e)+n_{0}(e) / 2\right) \times\left(n_{v}(e)+n_{0}(e) / 2\right)\right], \\
S z_{e}^{*}(G) & =\sum_{e=u v}\left[\left(m_{u}(e)+m_{0}(e) / 2\right) \times\left(m_{v}(e)+m_{0}(e) / 2\right)\right] .
\end{aligned}
$$

It is worth mentioning here that the Szeged index was introduced by Ivan Gutman [4] and the name Szeged index was given in [5]. For the mathematical properties of this topological index we refer to [3, 9, 10]. The concept of edge Szeged index was introduced in [6] and mathematical properties of this graph invariant are studied in $[2,7,8]$. The revised Szeged index was introduced by Milan Randić [13] as a modification of the classical Wiener index. Nowadays the scientists prefer the name revised Szeged index for this distance-based topological index. The interested readers can consult $[1,11,12,15]$ for mathematical properties of this new topological index.

Throughout this section graph means finite simple connected graph. The notation is standard and can be taken from the standard books on graph theory.

\section{Main results}

In this section, we first present a new formula for computing revised Szeged index of graphs. Then apply this new formula to reprove all results given by Aouchiche and Hansen [1]. We also present an edge version of the revised Szeged index and extend the results given in the mentioned article to this new graph invariant. We begin by an example.

Example 2.1. Suppose $G_{1}=K_{n}, G_{2}=C_{n}$ and $G_{3}=W_{n}$ denote the complete, cycle and wheel graphs of order $n$, and $G_{4}=K_{m, n}$ is the complete bipartite graph with partitions of size $m$ and $n$, respectively. Then,

- If $e=u v \in E\left(G_{1}\right)$ then $m_{u}=m_{v}=n-2$ and $m_{0}=\frac{n^{2}-5 n+8}{2}$. Therefore, $S z_{e}\left(G_{1}\right)=\frac{n(n-1)(n-2)^{2}}{2}$ and $S z_{e}^{*}\left(G_{1}\right)=\frac{n^{3}(n-1)^{3}}{32}$.

- Suppose $e=u v$ is an arbitrary edge of $G_{2}$. If $n=2 k+1$, then $m_{u}=m_{v}=k$ and so $m_{0}=1$. Therefore, $S z_{e}\left(G_{2}\right)=(2 k+1) k^{2}=\frac{n(n-1)^{2}}{4}$ and $S z_{e}^{*}\left(G_{2}\right)=\frac{n^{3}}{4}$. 
If $n=2 k$ then $m_{u}=m_{v}=k-1$ and so $m_{0}=2$. This implies that $S z_{e}\left(G_{2}\right)=$ $n(k-1)^{2}=\frac{n(n-2)^{2}}{4}$ and $S z_{e}^{*}\left(G_{2}\right)=\frac{n^{3}}{4}$.

- Consider the $n$-vertex wheel graph $G_{3}, n>5$. If $e=u v$ is an edge of $G_{3}$ such that the vertex $v$ is the center of $G_{3}$, then $m_{u}=3, m_{v}=2 n-7$ and $m_{0}=3$. If both of $u$ and $v$ are not the center of $G_{3}$, then $m_{u}=3, m_{v}=3$ and $m_{0}=2 n-8$. Therefore $S z_{e}\left(G_{3}\right)=(n-1)(4 n-5)$ and $S z_{e}^{*}\left(G_{3}\right)=(n-1)\left(n^{2}+5 n-73 / 4\right)$.

- Suppose $G_{4}=K_{x, y}, x+y=n$, is the complete bipartite graph containing an arbitrary edge $e=u v$, where $\operatorname{deg}(u)=x$ and $\operatorname{deg}(v)=y$. Then we have $m_{u}=$ $x-1, m_{v}=y-1, m_{0}=x y-x-y+2$. This implies that $S z_{e}\left(G_{4}\right)=x y(x-1)(y-1)$ and $S z_{e}^{*}\left(G_{4}\right)=\frac{x y}{4}\left(x^{2} y^{2}-x^{2}-y^{2}+2 x y\right)$.

Theorem 2.2. Let $G$ be an $n$-vertex and $m$-edge graph. Then

$$
S z^{*}(G)=\frac{m n^{2}}{4}-\frac{1}{4} \sum_{e=u v}\left(n_{u}^{2}+n_{v}^{2}\right)+\frac{1}{2} S z(G)
$$

Proof. Since $n_{u}(e)+n_{v}(e)=n-n_{0}(e)$ we have:

$$
\begin{aligned}
S z^{*}(G) & =\sum_{e=u v}\left[\left(n_{u}+\frac{n_{0}}{2}\right)\left(n_{v}+\frac{n_{0}}{2}\right)\right] \\
& =\sum_{e=u v}\left[n_{u} n_{v}+\frac{n_{0}}{2}\left(n_{u}+n_{v}\right)+\frac{1}{4} n_{0}^{2}\right] \\
& =\sum_{e=u v}\left[n_{u} n_{v}+\frac{1}{2}\left(\left(n-\left(n_{u}+n_{v}\right)\right)\right)\left(n_{u}+n_{v}\right)+\frac{1}{4}\left(n-\left(n_{u}+n_{v}\right)\right)^{2}\right] \\
& =\sum_{e=u v}\left[n_{u} n_{v}+\frac{n}{2}\left(n_{u}+n_{v}\right)-\frac{1}{2}\left(n_{u}+n_{v}\right)^{2}\right. \\
& =\sum_{e=u v}\left[\frac{n^{2}}{4}-\frac{1}{2} n\left(n_{u}+n_{v}\right)+\frac{1}{4}\left(n_{u}+n_{v}\right)^{2}\right] \\
= & \left.\frac{1}{2} S z(G)+\frac{m n^{2}}{4}-\frac{1}{4}\left(n_{u}^{2}+n_{v}^{2}+2\left(n_{u}\right)\left(n_{v}\right)\right)\right]
\end{aligned}
$$

proving the result.

The next Corollary is already known result that stated and proven in [1].

Corollary 2.3. $S z(G) \leq S z^{*}(G) \leq \frac{m n^{2}}{4}$.

Proof. Since $n_{u}+n_{v} \leq n,\left(n_{u}+n_{v}\right)^{2} \leq n^{2}$. So, $\sum_{e=u v}\left[n_{u}+n_{v}\right]^{2} \leq m n^{2}$ and therefore $\frac{m n^{2}}{4}-\frac{1}{4} \sum_{e=u v}\left[n_{u}+n_{v}\right]^{2}=\frac{m n^{2}}{4}-\frac{1}{4} \sum_{e=u v}\left[n_{u}^{2}+n_{v}^{2}\right]-\frac{1}{2} S z(G) \geq 0$. Now, Theorem 2.2 implies that $S z(G) \leq S z^{*}(G)$, the left hand side of inequality. The right hand side is a direct consequence of Theorem 2.2 and the following inequality:

$$
\frac{1}{2} S z(G)-\frac{1}{4} \sum_{e=u v}\left[n_{u}^{2}+n_{v}^{2}\right]=-\frac{1}{4} \sum_{e=u v}\left[n_{u}-n_{v}\right]^{2} \leq 0 .
$$


By a similar argument as Theorem 2.2, one can prove:

Theorem 2.4. Let $G$ be an n-vertex and m-edge graph. Then

$$
S z_{e}^{*}(G)=\frac{m^{3}}{4}-\frac{1}{4} \sum_{e=u v}\left[m_{u}^{2}+m_{v}^{2}\right]+\frac{1}{2} S z_{e}(G) .
$$

Corollary 2.5. $S z_{e}(G) \leq S z_{e}^{*}(G) \leq \frac{m^{3}}{4}$.

Proof. The proof is similar to the proof of Corollary 2.3 and so omitted.

Suppose $G$ is a connected graph and $u$ is a vertex of $G$. Define

$$
D(u, G)=\sum_{x \in V(G)}\left[d_{G}(u, x)\right]
$$

The graph $G$ is called distance-balanced (or transmission-regular according to [1]) if for every $u, v \in V(G), D(u, G)=D(v, G)$. Similarly for a vertex $u$ and an edge $e=x y$ define $D_{e}(u, G)=\sum_{e \in E(G)}\left[d_{G}(e, u)\right.$. A graph $G$ is called edge-distance-balanced if for every vertices $u, v \in V(G), D_{e}(u, G)=d_{e}(v, G)$.

Theorem 2.6. Suppose $u$ and $v$ are vertices of a connected graph $G$. Then $m_{u}=m_{v}$ if and only if $D_{e}(u, G)=d_{e}(v, G)$.

Proof. Let $e=u v$ be an arbitrary edge of $G$. We partition the edge set of $G$ into three parts as follows:

- $M(u)$ is the set of all edges that are closer to $u$ than $\mathrm{v}$.

- $M(v)$ is the set of all edges that are closer to $v$ than $u$.

- $M(o)$ is the set of all edges that are equidistant from $u$ and $v$.

Suppose $m_{u}(e)=|M(u)|, m_{v}(e)=|M(v)|$ and $m_{0}(e)=|M(o)|$. Then we have :

$$
\begin{aligned}
D_{e}(u, G) & =\sum_{e \in E(G)} d_{G}(e, u) \\
& =\sum_{e \in M(u)} d_{G}(e, u)+\sum_{e \in M(v)} d_{G}(e, u)+\sum_{e \in M(0)} d_{G}(e, u) \\
& =\sum_{e \in M(u)} d_{G}(e, u)+\sum_{e \in M(v)}\left(1+d_{G}(e, v)\right)+\sum_{e \in M(0)} d_{G}(e, u) \\
& =\sum_{e \in M(u)} d_{G}(e, u)+m_{v}(e)+\sum_{e \in M(v)} d_{G}(e, v)+\sum_{e \in M(0)} d_{G}(e, u) .
\end{aligned}
$$

A similar argument shows that

$$
D_{e}(v, G)=\sum_{e \in M(u)} d_{G}(e, u)+m_{u}(e)+\sum_{e \in M(v)} d_{G}(e, v)+\sum_{e \in M(0)} d_{G}(e, v) .
$$

But $D_{e}(u, G)-D_{e}(v, G)=m_{v}(e)-m_{u}(e)$ and so $m_{u}(e)=m_{v}(e)$ if and only if $D_{e}(u, G)=D_{e}(v, G)$. This complete our argument. 
Corollary 2.7. If $S z_{e}(G)=\frac{m^{3}}{4}$ then $G$ is an edge-distance-balanced graph.

Proof. If $S z_{e}(G)=\frac{m^{3}}{4}$ then by Corollary $2.5, S z_{e}^{*}(G)=\frac{m^{3}}{4}$. Thus

$$
\frac{1}{2} S z_{e}(G)-\frac{1}{4} \sum_{u v \in E(G)}\left[m_{u}^{2}+m_{v}^{2}\right]=-\frac{1}{4} \sum_{u v \in E(G)}\left[m_{u}-m_{v}\right]^{2}=0 .
$$

Therefore $m_{u}=m_{v}$. Now Theorem 2.6 implies that $G$ is an edge-distanced-balanced graph.

In the end of this paper, we compute an exact formula for the edge revised Szeged index of Cartesian product of graphs. To do this, we assume that $G$ and $H$ are connected graphs with vertex sets $V(G)=\left\{u_{1}, u_{2}, \ldots, u_{r}\right\}$ and $V(H)=\left\{v_{1}, v_{2}, \ldots, v_{s}\right\}$. We also assume that $|E(G)|=e_{1}$ and $|E(H)|=e_{2}$. Then by definition $V(G \times H)=V(G) \times V(H)$ and we have:

$$
E(G \times H)=\{(u, v)(a, b) \mid[u=a, v b \in E(H)] \text { or }[u a \in E(G), v=b]\} .
$$

Clearly, $|E(G \times H)|=|V(G)||E(H)|+|V(H)||E(G)|$. To compute the edge revised Szeged index of $G \times H$ we partition the edge set of this graph into the following parts:

$$
\begin{aligned}
A_{m} & =\left\{\left(u_{m}, x\right)\left(u_{m}, y\right) \mid x y \in E(H)\right\} ; 1 \leq m \leq r, \\
B_{t} & =\left\{\left(a, v_{t}\right)\left(b, v_{t}\right) \mid a b \in E(G)\right\} ; 1 \leq t \leq s .
\end{aligned}
$$

Theorem 2.8. (See [15, Lemmas 2 and 3]). With above notations we have:

(a) If $\left.e=\left(u_{m}, v_{j}\right)\left(u_{m}, v_{q}\right) \in A_{m}\right)$ then

$$
\begin{aligned}
& m_{\left(u_{m}, v_{j}\right)}(e)=|V(G)| m_{v_{j}}\left(v_{j} v_{q}\right)+|E(G)| n_{v_{j}}\left(v_{j} v_{q}\right)=r m_{v_{j}}(H)+e_{1} n_{v_{j}}(H), \\
& m_{\left(u_{m}, v_{q}\right)}(e)=|V(G)| m_{v_{q}}\left(v_{j} v_{q}\right)+|E(G)| n_{v_{j}}\left(v_{j} v_{q}\right)=r m_{v_{q}}(H)+e_{1} n_{v_{q}}(H) .
\end{aligned}
$$

(b) If $e=\left(u_{i}, v_{t}\right)\left(u_{p}, v_{t}\right) \in B_{t}$ then

$$
\begin{gathered}
m_{\left(u_{i}, v_{t}\right)}(e)=|V(H)| m_{u_{i}}\left(u_{i} u_{p}\right)+|E(H)| n_{u_{i}}\left(u_{i} u_{p}\right)=s m_{u_{i}}(G)+e_{2} n_{u_{i}}(G), \\
m_{\left(u_{p}, v_{t}\right)}(e)=|V(H)| m_{u_{p}}\left(u_{i} u_{p}\right)+|E(H)| n_{u_{p}}\left(u_{i} u_{p}\right)=s m_{u_{p}}(G)+e_{2} n_{u_{p}}(G) .
\end{gathered}
$$

Theorem 2.9. With notation of Theorem 2.8, the edge revised Szeged index of Cartesian product of $G$ and $H$ can be computed as follows:

$$
\begin{aligned}
S z_{e}^{*}(G \times H) & =\frac{1}{2} r^{3} S z_{e}(H)+r^{2} e_{1} S z_{e v}(H)+\frac{1}{2} r e_{1}^{2} S z(H)+\frac{1}{4} r e_{2}\left(r e_{2}+s e_{1}\right)^{2} \\
& -r^{2} e_{1} S z_{v e}(H)-\frac{1}{4} r^{3} \sum_{x y \in E(H)}\left[m_{x}^{2}(H)+m_{y}^{2}(H)\right] \\
& -\frac{1}{4} r e_{1}^{2} \sum_{x y \in E(H)}\left[n_{x}^{2}(H)+n_{y}^{2}(H)\right]+\frac{1}{2} s^{3} S z_{e}(G)+s^{2} e_{2} S z_{e v}(G) \\
& +\frac{1}{2} s e_{2}^{2} S z(G)+\frac{1}{4} s e_{1}\left(s e_{1}+r e_{2}\right)^{2}-s^{2} e_{2} S z_{v e}(G) \\
& -\frac{1}{4} s^{3} \sum_{a b \in E(G)}\left[m_{a}^{2}(G)+m_{b}^{2}(G)\right]-\frac{1}{4} s e_{2}^{2} \sum_{a b \in E(G)}\left[n_{a}^{2}(G)+n_{b}^{2}(G)\right] .
\end{aligned}
$$


Proof. Let $e=\left(u_{m}, x\right)\left(u_{m}, y\right) \in A_{m}$. Then $m_{0}(e)=r e_{2}+s e_{1}-r\left(m_{x}(H)+m_{y}(H)\right)$ $-e_{1}\left(n_{x}(H)+n_{y}(H)\right)$. Set,

$$
\begin{aligned}
& A=\left[m_{\left(u_{m}, x\right)}(e)+\frac{m_{0}(e)}{2}\right] \times\left[m_{\left(u_{m}, y\right)}(e)+\frac{m_{0}(e)}{2}\right], \\
& B=\left[m_{\left(a, v_{t}\right)}(e)+\frac{m_{0}(e)}{2}\right] \times\left[m_{\left(b, v_{t}\right)}(e)+\frac{m_{0}(e)}{2}\right] .
\end{aligned}
$$

Then we have:

$$
\begin{aligned}
A & =\frac{1}{2} r^{2} m_{x}(H) m_{y}(H)+\frac{1}{2} e_{1} r\left(n_{x}(H) m_{y}(H)+n_{y}(H) m_{x}(H)\right) \\
& +\frac{1}{2} e_{1}^{2} n_{x}(H) n_{y}(H)+\frac{1}{4}\left(r e_{2}+s e_{1}\right)^{2}-\frac{1}{2} e_{1} r\left(n_{x}(H) m_{x}(H)+n_{y}(H) m_{y}(H)\right) \\
& -\frac{1}{4} r^{2}\left(m_{x}^{2}(H)+m^{2} y(H)\right)-\frac{1}{4} e_{1}^{2}\left(n_{x}^{2}(H)+n_{y}^{2}(H)\right) .
\end{aligned}
$$

Thus,

$$
\begin{aligned}
\sum_{\left(u_{m}, x\right)\left(u_{m}, y\right) \in A_{m}} & {\left[m_{\left(u_{m}, x\right)}(e)+\frac{m_{o}(e)}{2}\right]\left[m_{\left(u_{m}, y\right)}(e)+\frac{m_{0}(e)}{2}\right] } \\
& =1 / 2 r^{2} S z_{e}(H)+e_{1} r S z_{e v}(H)+\frac{1}{2} e_{1}^{2} S z(H)+\frac{1}{4} e_{2}\left(r e_{2}+s e_{1}\right)^{2} \\
& -e_{1} r S z_{v e}(H)-\frac{1}{4} r^{2} \sum_{x y \in E(H)}\left[m_{x}^{2}(H)+m_{y}^{2}(H)\right] \\
& -\frac{1}{4} e_{1}^{2} \sum_{x y \in E(H)}\left[n_{x}^{2}(H)+n_{y}^{2}(H)\right] .
\end{aligned}
$$

Using a similar argument for the edge $e=\left(a, v_{t}\right)\left(b, v_{t}\right) \in B_{t}$, we have:

$$
\begin{aligned}
B & =\left[m_{\left(a, v_{t}\right)}(e)+\frac{m_{0}(e)}{2}\right]\left[m_{\left(b, v_{t}\right)}(e)+\frac{m_{0}(e)}{2}\right] \\
& =\frac{1}{2} s^{2} m_{u_{i}}(G) m_{u_{p}}(G)+\frac{1}{2} e_{2} s\left[n_{a}(G) m_{b}(G)+n_{b}(G) m_{a}(G)\right] \\
& +\frac{1}{2} e_{2}^{2} n_{a}(G) n_{b}(G)+\frac{1}{4}\left(s e_{1}+r e_{2}\right)^{2}-\frac{1}{2} e_{2} s\left[n_{a}(G) m_{a}(G)+n_{b}(G) m_{b}(G)\right] \\
& -\frac{1}{4} s^{2}\left[m_{a}^{2}(G)+m_{b}^{2}(G)\right]-\frac{1}{4} e_{2}^{2}\left[n_{a}^{2}(G)+n_{b}^{2}(G)\right] .
\end{aligned}
$$

So,

$$
\begin{aligned}
\sum_{\left(a, v_{t}\right)\left(b, v_{t}\right) \in B_{t}} & {\left[m_{\left(a, v_{t}\right)}(e)+\frac{m_{0}(e)}{2}\right]\left[m_{\left(b, v_{t}\right)}(e)+\frac{m_{0}(e)}{2}\right] } \\
= & \frac{1}{2} s^{2} S z_{e}(G)+e_{2} s S z_{e v}(G)+\frac{1}{2} e_{2}^{2} S z(G)+\frac{1}{4} e_{1}\left(s e_{1}+r e_{2}\right)^{2} \\
& -e_{2} s S z_{v e}(G)-\frac{1}{4} s^{2} \sum_{a b \in E(G)}\left[m_{a}^{2}(G)+m_{b}^{2}(G)\right] \\
& -\frac{1}{4} e_{2}^{2} \sum_{a b \in E(G)}\left[n_{a}^{2}(G)+n_{b}^{2}(G)\right]
\end{aligned}
$$


Now multiplying Eq. (2.1) by $r$ and Eq. (2.2) by $s$ and summation of these values, the formula given in the theorem will be obtained.

\section{Conclusions}

Some of mathematicians recently focus on the revised Szeged index of graphs. In this paper a new formula for computing this topological index is presented by which it is possible to reprove some earlier results. We also investigate an edge version of this interesting topological index. We proved that the edge version of this graph invariant is more complicated than its vertex version. In the case of vertex version, it is easy to find an exact formula for the Cartesian product of graphs but in the edge version it is too difficult.

In Theorem 2.6 and Corollary 2.7, it is proved that $S z_{e}(G)=\frac{m^{3}}{4}$ implies that $G$ is an edge-balanced-distance graph. We end the paper with the following open question:

Question: Characterize graphs $G$ such that $S z_{e}(G)=m^{3} / 4$.

\section{Acknowledgements}

The authors are indebted to the referees for their suggestions and helpful remarks. This research has been supported by the research affair of the University of Kashan, I. R. Iran under grant number 159020/4.

\section{References}

[1] M. Aouchiche and P. Hansen, On a conjecture about the Szeged index, European J. Combin. 31 (2010), 1662-1666.

[2] E. Chiniforooshan and B. Wu, Maximum values of Szeged index and edge- Szeged index of graphs, Electronic Notes Discrete Math. 34 (2009), 405-409.

[3] K. Ch. Das and I. Gutman, Estimating the Szeged index, Appl. Math. Lett. 22 (2009), 16801684.

[4] I. Gutman, A formula for the Wiener number of trees and its extension to graphs containing cycles, Graph Theory Notes New York 27 (1994), 9-15.

[5] I. Gutman, P. V. Khadikar, P. V. Rajput and S. Karmarkar, The Szeged index of polyacenes, J. Serb. Chem. Soc. 60 (1995), 759-764.

[6] I. Gutman and A. R. Ashrafi, The edge version of the Szeged index, Croat. Chem. Acta 81 (2008), 277-281.

[7] M. H. Khalifeh, H. Yousefi-Azari, A. R. Ashrafi and I. Gutman, The edge Szeged index of product graphs, Croat. Chem. Acta 81 (2008), 277-281.

[8] M. H. Khalifeh, H. Yousefi-Azari, A. R. Ashrafi and S. G. Wagner, Some new results on distance-based graph invariants, European J. Combin. 30 (2009), 1149-1163.

[9] S. Klavžar and I. Gutman, A theorem on Wiener-type invariants for isometric subgraphs of hypercubes, Appl. Math. Lett. 19 (2006), 1129-1133.

[10] T. Mansour and M. Schork, The vertex PI index and Szeged index of bridge graphs, Discrete Appl. Math. 157 (2009), 1600-1606.

[11] T. Pisanski and M. Randić, Use of the Szeged index and the revised Szeged index for measuring network bipartivity, Discrete Appl. Math. 158 (2010), 1936-1944. 
[12] T. Pisanski and J. Žerovnik, Edge-contributions of some topological indices and arboreality of molecular graphs, Ars Mathematica Contemporanea 2 (2009), 49-58.

[13] M. Randić, On generalization of Wiener index for cyclic structures, Acta Chim. Slovenica 49 (2002), 483-496.

[14] H. Wiener, Structural determination of paraffin boiling points, J. Am. Chem. Soc. 69 (1947), $17-20$.

[15] R. Xing and B. Zhou, On the revised Szeged index, Discrete Appl. Math. 159 (2011), 69-78. 